Review began 10/11/2021 Review ended 10/30/2021 Published 10/31/2021

() Copyright 2021

Oike et al. This is an open access article distributed under the terms of the Creative Commons Attribution License CC-BY 4.0., which permits unrestricted use, distribution, and reproduction in any medium, provided the original author and source are credited.

\title{
Radiotherapy With Hydrogen Peroxide-Soaked Gauze for Unresectable Breast Cancer
}

\author{
Takahiro Oike $^{1,2}$, Kento Tomizawa ${ }^{3}$, Akiko Adachi ${ }^{2,3}$, Masahiro Wada ${ }^{4}$, Tatsuya Ohno ${ }^{3,1}$ \\ 1. Heavy Ion Medical Center, Gunma University, Maebashi, JPN 2. Department of Radiation Oncology, Sano Kousei \\ General Hospital, Sano, JPN 3. Department of Radiation Oncology, Gunma University Graduate School of Medicine, \\ Maebashi, JPN 4. Department of Breast Surgery, Sano Kousei General Hospital, Sano, JPN
}

Corresponding author: Takahiro Oike, oiketakahiro@gmail.com

\begin{abstract}
A hydrogen peroxide $\left(\mathrm{H}_{2} \mathrm{O}_{2}\right)$-soaked gauze has been used in combination with radiotherapy in anticipation of sensitizing tumors exposed to the skin surface. Although used empirically in the clinic, the method is rarely reported in the literature, making its efficacy and tolerability unclear. Here, we report a case of primary metastatic breast cancer whose primary tumor was treated with palliative radiotherapy using an $\mathrm{H}_{2} \mathrm{O}_{2}$-soaked gauze. The primary tumor in the right breast regrew after treatment with palbociclib plus letrozole followed by fulvestrant and denosumab. The tumor was exposed to the skin surface, causing exudation, bleeding, pain, and difficulty in raising the right upper limb. Radiotherapy (51 Gy in 17 fractions) using the $\mathrm{H}_{2} \mathrm{O}_{2}$-soaked gauze resolved the patient's symptoms and the tumor showed macroscopic complete remission at three months post-treatment. This case indicates that radiotherapy with an $\mathrm{H}_{2} \mathrm{O}_{2}$-soaked gauze is an effective and tolerable palliative treatment for superficially exposed tumors. This non-invasive and inexpensive method of radiosensitization warrants validation and optimization in a prospective setting.
\end{abstract}

Categories: Radiation Oncology

Keywords: bleeding, hydrogen peroxide, palliation, breast cancer, radiotherapy

\section{Introduction}

Radiotherapy is used to treat tumors in frail or elderly individuals who are not suitable for or refuse surgery. However, it is often difficult for radiotherapy alone to eradicate tumors or even to relieve tumor-related symptoms such as bleeding and pain. Thus, there is an unmet clinical need for novel methods of radiosensitization.

Preclinical evidence suggests that hydrogen peroxide $\left(\mathrm{H}_{2} \mathrm{O}_{2}\right)$ sensitizes cancer cells to X-rays [1,2]. A study in a mouse tumor model showed that intratumoral injection of $0.5 \% \mathrm{H}_{2} \mathrm{O}_{2}$ (in $0.83 \%$ sodium hyaluronate gel) combined with X-rays resulted in greater growth suppression than X-rays alone [3]. A recent phase one clinical trial analyzing 12 inoperable patients with breast cancer demonstrated that intratumoral injection of $\mathrm{H}_{2} \mathrm{O}_{2}$ plus radiotherapy was tolerable [4]. Based on these data, a phase two clinical trial is underway.

On the other hand, an $\mathrm{H}_{2} \mathrm{O}_{2}$-soaked gauze has been used in combination with radiotherapy in anticipation of sensitizing the tumors exposed to the skin surface. Although this method is used empirically in the clinic, the use of an $\mathrm{H}_{2} \mathrm{O}_{2}$-soaked gauze is rarely reported in the literature (in contrast to intratumoral injection of $\mathrm{H}_{2} \mathrm{O}_{2}$ ); thus, the efficacy and tolerability of the bolus method are unclear. Here, we report a case of primary metastatic breast cancer whose primary tumor was treated with palliative radiotherapy plus an $\mathrm{H}_{2} \mathrm{O}_{2}$-soaked gauze.

\section{Case Presentation}

A 77-year-old woman was referred to our radiation oncology unit for palliative treatment of a recurrent primary tumor in the right breast. The tumor caused exudation, bleeding, pain, and difficulty in raising the right upper limb, thereby reducing the patient's quality of life (QOL).

Nineteen months before the referral, the patient visited the surgical oncology unit and was newly diagnosed with stage IV (T2N0M1) breast cancer with lung metastases. The primary tumor occupied the lateral half of the right breast. The tumor was $47 \mathrm{~mm}$ in diameter, as assessed by computed tomography (CT), and was estrogen and progesterone receptor-positive, and human epidermal growth factor receptor-2 (HER2)negative. The patient developed bone metastasis after four courses of palbociclib plus letrozole as first-line treatment. After 13 courses of fulvestrant as second-line treatment combined with monthly denosumab, the stable primary tumor became progressive, with axially lymph node metastasis (Figure 1). The primary tumor was considered inoperable, resulting in a referral to the radiation oncology unit. 


\section{Cureus}
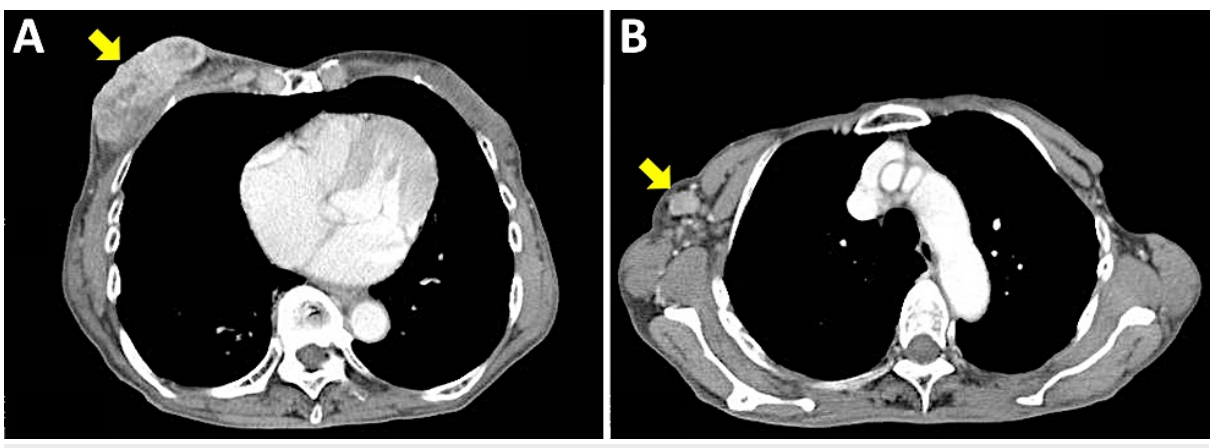

FIGURE 1: Contrast-enhanced computed tomography image obtained at the time of referral to the radiation oncology unit.

(A) The primary tumor in the right breast $(70 \times 24 \mathrm{~mm})$. (B) Metastasis to a right axial lymph node $(15 \times 11 \mathrm{~mm})$. Arrows indicate the tumors.

Employing standard tangentially opposed irradiation techniques, we performed conventional threedimensional conformal radiotherapy with 6-MV photons [4]. The gross tumor, metastatic node, and tumor bed received 51 Gy delivered in 17 fractions (five fractions per week) (Figure 2). At each radiotherapy session, an $\mathrm{H}_{2} \mathrm{O}_{2}$-soaked gauze (approximately $5 \mathrm{~mm}$ thick) was freshly prepared and placed on the right breast to cover the tumor (Figure 3). Practically, when the patient entered the treatment room, a nurse poured oxydol (i.e., 2.5-3.5 w/v\% $\mathrm{H}_{2} \mathrm{O}_{2}$; KENEI Pharmaceutical Co. Ltd., Osaka, Japan) in a plastic bag and a gauze was soaked in the bag. As the treatment progressed, the tumor showed a fair amount of shrinkage. Exudation and bleeding from the tumor, as well as the pain and difficulty in raising the right upper limb, resolved upon completion of the treatment. The patient experienced grade one radiation dermatitis (assessed by the Common Terminology Criteria for Adverse Events 4.0) but did not experience pain from the application of the $\mathrm{H}_{2} \mathrm{O}_{2}$ gauze. The dermatitis was resolved post-radiotherapy without topical medication.
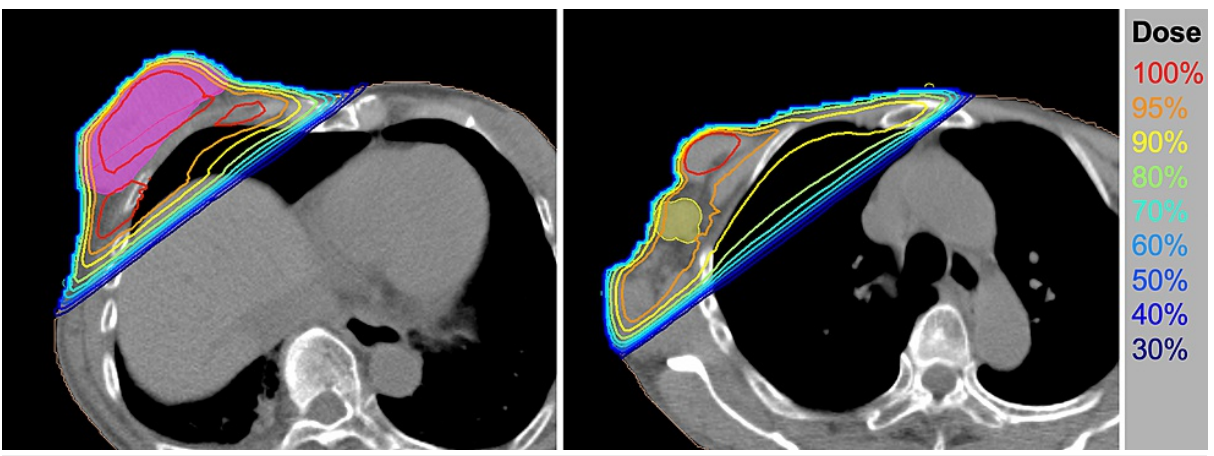

FIGURE 2: Treatment plan for radiotherapy.

(A) A plane showing the primary tumor (solid magenta). (B) A plane showing the axial lymph node metastasis (solid yellow). Isodose lines are shown on computed tomography images. 


\section{Cureus}

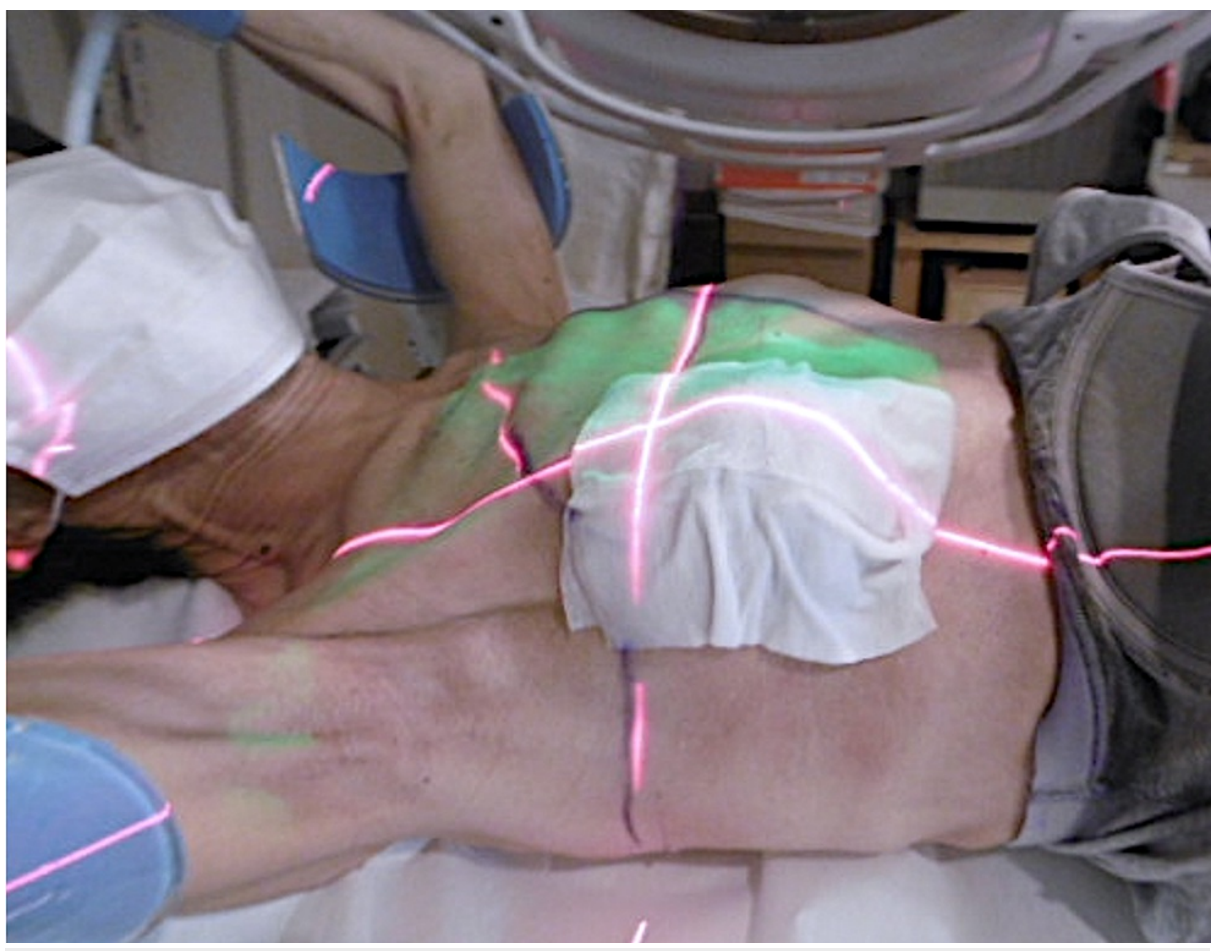

FIGURE 3: Application of the hydrogen peroxide-soaked gauze during the radiotherapy session.

Upon completion of radiotherapy, tegafur was started as a third-line treatment. At three months postcompletion of radiotherapy, the tumor showed macroscopic complete remission (Figure 4). The irradiated tumor was almost unidentifiable in CT images obtained at four months post-completion of radiotherapy (Figure 5). Serum cancer antigen 15-3 (CA15-3), a marker reflecting disease activity, also showed a substantial decrease after radiotherapy (Figure 6 ). The patient has resumed her daily life without symptoms and with peace of mind, although the progressive disease is present in other organs. 


\section{Cureus}
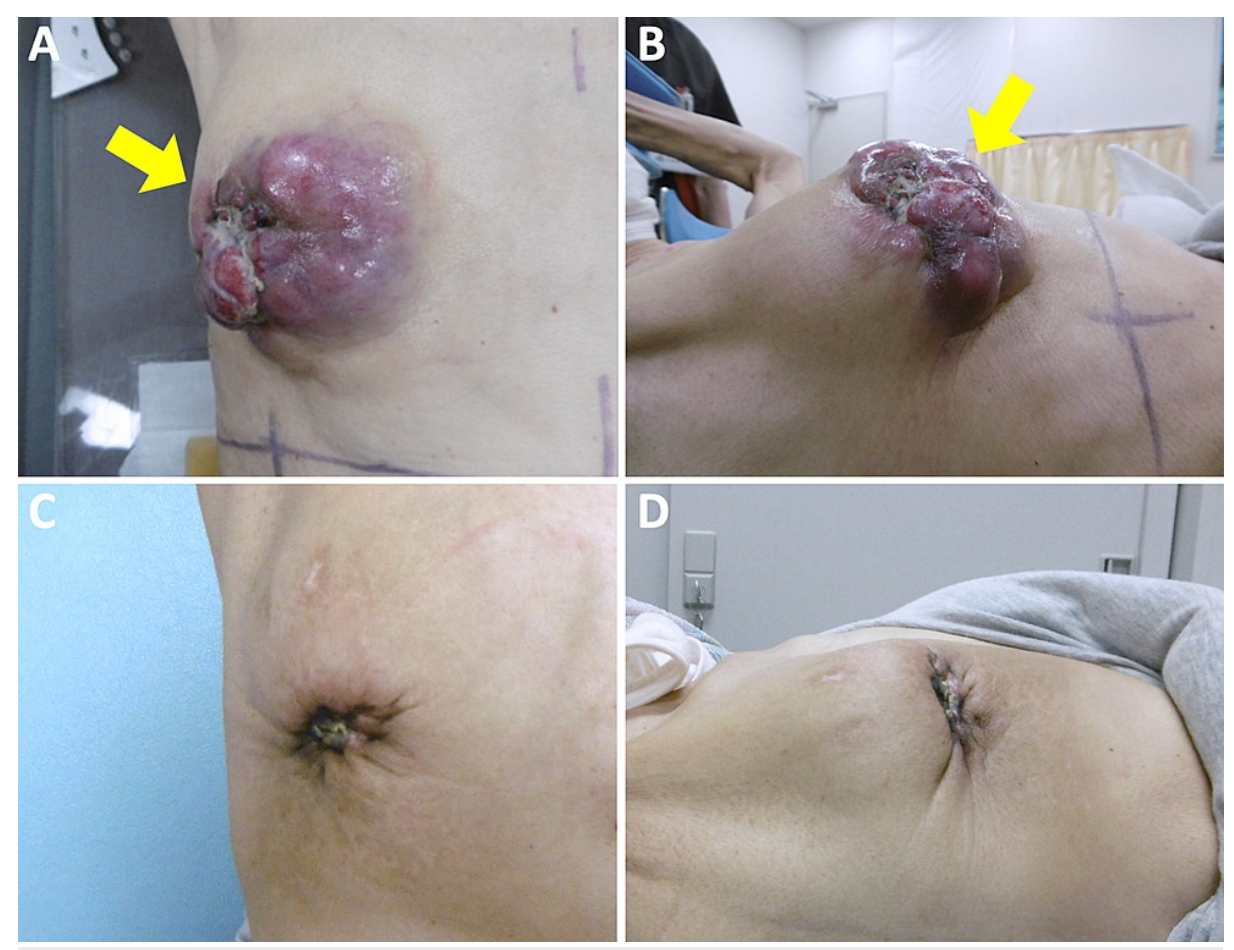

FIGURE 4: Pictures of the patient's right breast.

(A, B) Radiotherapy day one. Arrows indicate the primary tumor. (C, D) Three months post-completion of radiotherapy.

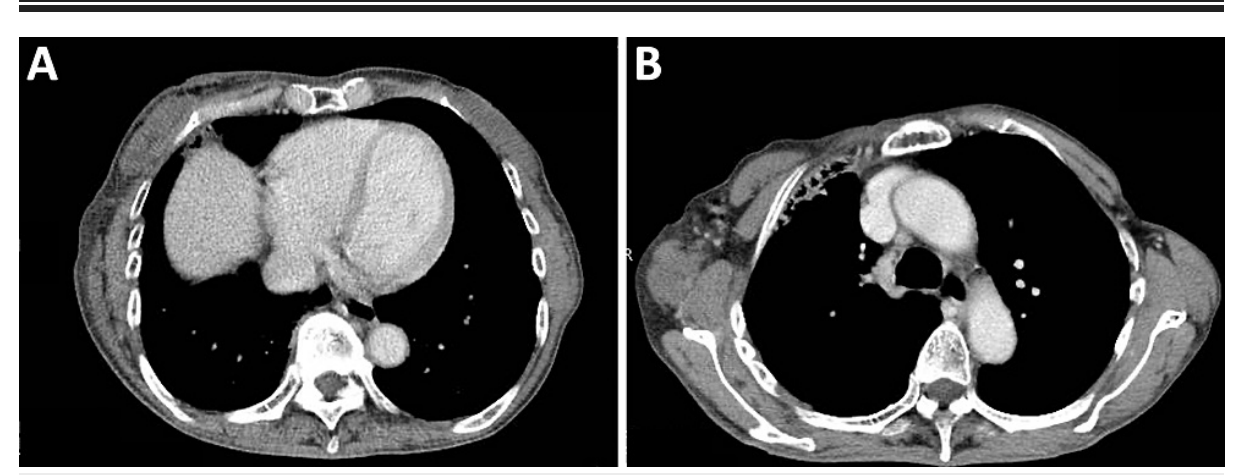

FIGURE 5: Contrast-enhanced computed tomography image obtained at four months post-treatment.

(A) Primary tumor site. (B) Metastatic lymph node site. 


\section{Cureus}

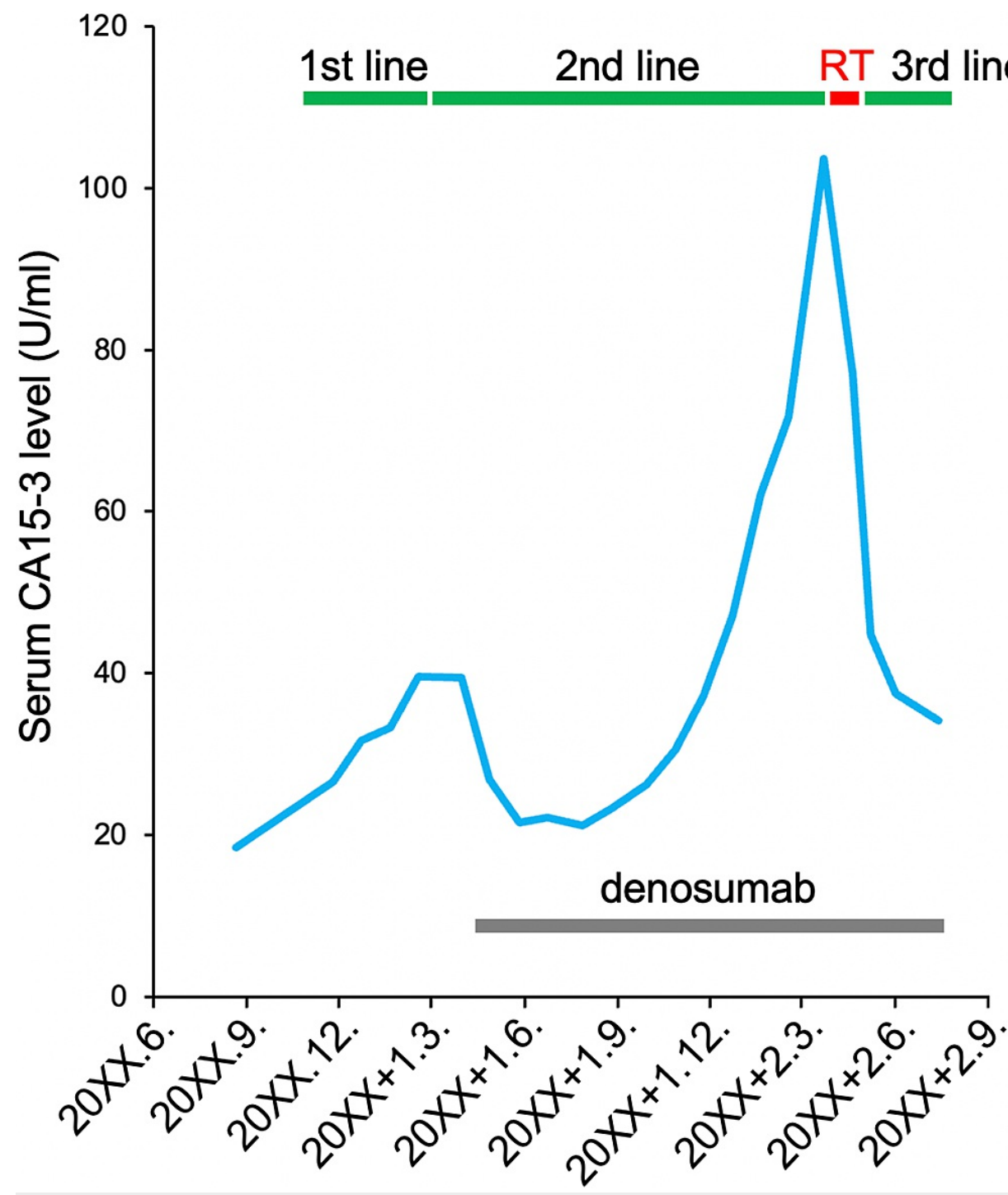

FIGURE 6: Serum CA15-3 kinetics.

1st line, palbociclib plus letrozole; 2nd line, fulvestrant; 3rd line, tegafur; RT, radiotherapy with the hydrogen peroxide bolus; CA15-3, cancer antigen 15-3.

\section{Discussion}

Breast cancer exposed to the skin surface threatens QOL severely by causing various symptoms including bleeding and pain. Such cases are often medically inoperable. In addition, clinical evidence suggests that, at least for breast cancer, approximately half of the tumor volume is hypoxic; hypoxia-enriched tumors are radioresistant [5]. Therefore, radiotherapy alone may be insufficient to resolve the symptoms, especially in the case of bulky tumors. In our case, radiotherapy with an $\mathrm{H}_{2} \mathrm{O}_{2}$-soaked gauze led to temporal complete remission of a bulky breast tumor exposed to the skin surface, with minimal toxicity; these findings suggest the potential efficacy and tolerability of this treatment for palliation.

The radiosensitization strategy using an $\mathrm{H}_{2} \mathrm{O}_{2}$-soaked gauze was first described by Ogawa and colleagues in 2008 [6]. In that paper, the authors reported five cases of locally advanced and/or recurrent tumors exposed to the skin surface, which were treated with electrons plus an $\mathrm{H}_{2} \mathrm{O}_{2}$-soaked gauze (Table 1). A few other cases treated with the same strategy have been reported in a Japanese book (Table 1). In the previously reported cases, the equivalent dose in $2 \mathrm{~Gy}$ per fraction with an $\alpha / \beta$ ratio of $10\left(\mathrm{EQD} 2_{\alpha / \beta 10}\right)$ ranged from 54 Gy to $64 \mathrm{~Gy}$. All cases showed a partial or complete response. A total of $85 \%(6 / 7)$ of the cases treated using EQD $2_{\alpha / \beta 10}$ equal to or less than $56 \mathrm{~Gy}$ experienced grade one radiation dermatitis, whereas one patient with penile cancer treated using $64 \mathrm{~Gy}$ in 32 fractions experienced grade three radiation dermatitis. In our case, the EQD2 $\alpha / \beta 10$ was $55.2 \mathrm{~Gy}$ and the treatment resulted in temporal complete tumor remission, with grade one radiation dermatitis. These data indicate that the use of an $\mathrm{H}_{2} \mathrm{O}_{2}$-soaked gauze is tolerable when 
administered alongside an EQD2 $\alpha / \beta 10$ of approximately $<60 \mathrm{~Gy}$. Nevertheless, solid evidence of tolerable and optimal dose fractionation for this treatment is still lacking, thereby warranting further research in a prospective setting.

\begin{tabular}{|c|c|c|c|c|c|c|c|c|}
\hline Cancer type & $\mathbf{n}$ & Radiation & Dose/fr. & $E Q D 2 \alpha / \beta 10$ & $\begin{array}{l}\text { Follow-up } \\
\text { (M) }\end{array}$ & Outcome & Adverse effect & Refs \\
\hline Breast cancer & 1 & Electrons & $48 \mathrm{~Gy} / 12 \mathrm{fr}$. & 56 Gy & 3 & PR & Dermatitis (G1) & [6] \\
\hline Melanoma & 1 & Electrons & $48 \mathrm{~Gy} / 12 \mathrm{fr}$. & 56 Gy & 12 & CR & Dermatitis (G1) & [6] \\
\hline $\begin{array}{l}\text { Malignant fibrous } \\
\text { histiocytoma }\end{array}$ & 1 & Electrons & $48 \mathrm{~Gy} / 12 \mathrm{fr}$. & 56 Gy & 3 & PR & Dermatitis (G1) & [6] \\
\hline $\begin{array}{l}\text { Skin squamous cell } \\
\text { carcinoma }\end{array}$ & 1 & Electrons & $48 \mathrm{~Gy} / 12 \mathrm{fr}$. & 56 Gy & 1 & PR & Dermatitis (G1) & [6] \\
\hline $\begin{array}{l}\text { Extramammary Paget's } \\
\text { disease }\end{array}$ & 1 & Electrons & $48 \mathrm{~Gy} / 12 \mathrm{fr}$. & 56 Gy & 12 & CR & Dermatitis (G1) & [6] \\
\hline Angiosarcoma & 2 & Electrons & $\begin{array}{l}52.5 \mathrm{~Gy} / 21 \\
\mathrm{fr} .\end{array}$ & $54.7 \mathrm{~Gy}$ & NA & NA & $\begin{array}{l}\text { Dermatitis (G2, } \\
50 \%)\end{array}$ & * \\
\hline Malignant trichilemmoma & 1 & X-rays & $54 \mathrm{~Gy} / 27 \mathrm{fr}$. & 54 Gy & 3 & CR & NA & * \\
\hline Penile cancer & 1 & X-rays & 64 Gy/32 fr. & 64 Gy & NA & CR & Dermatitis (G3) & * \\
\hline Breast cancer & 1 & X-rays & $51 \mathrm{~Gy} / 17 \mathrm{fr}$. & $55.2 \mathrm{~Gy}$ & 4 & CR & Dermatitis (G1) & $\begin{array}{l}\text { Present } \\
\text { case }\end{array}$ \\
\hline
\end{tabular}

TABLE 1: Summary of previously reported cases of superficial tumors treated with radiotherapy using a hydrogen peroxide bolus.

fr., fraction; EQD2 $\alpha / \beta 10$, the equivalent dose in 2 Gy per fraction with $\alpha / \beta$ of $10 ; M$, months; Refs, references; NA, not assessed; PR, partial response; CR, complete response. Adverse effect was assessed by the Common Terminology Criteria for Adverse Events 4.0, where $\mathrm{G}$ indicates grade. *Japanese book (ISBN: 978-4-86705-804-6).

Although the detailed mechanisms underlying the radiosensitizing effect of $\mathrm{H}_{2} \mathrm{O}_{2}$ are currently being investigated in the laboratory setting, the putative mechanism is as follows: first, two molecules of $\mathrm{H}_{2} \mathrm{O}_{2}$ break down to yield one molecule of oxygen and two molecules of water. This process can contribute to the reoxygenation of the radioresistant hypoxic tumor microenvironment. Second, $\mathrm{H}_{2} \mathrm{O}_{2}$ in combination with ionizing radiation induces intracellular production of reactive oxygen species (ROS). This can increase the induction of DNA double-strand breaks (i.e., the indirect effect). In addition, it is possible that ROS activate inflammatory signals that may upregulate antitumor immune responses [7]. Third, $\mathrm{H}_{2} \mathrm{O}_{2}$ influences a number of radioresistance-associated signaling pathways, including extracellular signal-regulated kinase (ERK), mitogen-activated protein kinase (MAPK), and nuclear factor kappa B (NFkB) [8-10]. Further research is needed to fully elucidate the underlying mechanisms and the optimal method of radiosensitization by $\mathrm{H}_{2} \mathrm{O}_{2}$.

As the limitation of this case report, first, there is a possibility that the $\mathrm{H}_{2} \mathrm{O}_{2}$-soaked gauze just functioned to improve dose distribution as same as water-soaked gauze. In fact, a water-density 5 -mm thick structure improved the dose distribution near the skin in this case (data not shown). To address this issue, a randomized study, that employs water-soaked gauze as control, is mandatory. Second, we used a commercially available oxydol (i.e., $2.53 .5 \mathrm{w} / \mathrm{v} \% \mathrm{H}_{2} \mathrm{O}_{2}$ ) according to the original report [6]; nevertheless, there is a possibility that the concentration of $\mathrm{H}_{2} \mathrm{O}_{2}$ can be further optimized so that it achieves maximal radiosensitizing effect with tolerable adverse effect, warranting further research. Third, in our case, we cannot exclude the possibility that tegafur contributed to the fair tumor response post-radiotherapy. Lastly, the possibility that the present case was an exceptional responder to ionizing radiations cannot be excluded, warranting validation by a prospective study.

\section{Conclusions}

An $\mathrm{H}_{2} \mathrm{O}_{2}$-soaked gauze has been used in combination with radiotherapy in anticipation of sensitizing the tumor exposed to the skin surface. Although this method is used empirically in the clinic, the use of an 
$\mathrm{H}_{2} \mathrm{O}_{2}$-soaked gauze is rarely reported in the literature (in contrast to intratumoral injection of $\mathrm{H}_{2} \mathrm{O}_{2}$ ); thus, the efficacy and tolerability of the gauze method are unclear. Here, we report a case of primary metastatic breast cancer whose primary tumor was treated with palliative radiotherapy plus an $\mathrm{H}_{2} \mathrm{O}_{2}$-soaked gauze.

Exudation, bleeding, pain, and difficulty in raising the right upper limb were resolved and the tumor showed macroscopic complete remission at three months post-treatment. This case indicates that radiotherapy with an $\mathrm{H}_{2} \mathrm{O}_{2}$-soaked gauze is both effective and tolerable as a palliative treatment for superficially exposed tumors.

\section{Additional Information \\ Disclosures}

Human subjects: Consent was obtained or waived by all participants in this study. Ethical Review Committee of Sano Kousei General Hospital issued approval Exempt. Conflicts of interest: In compliance with the ICMJE uniform disclosure form, all authors declare the following: Payment/services info: All authors have declared that no financial support was received from any organization for the submitted work. Financial relationships: All authors have declared that they have no financial relationships at present or within the previous three years with any organizations that might have an interest in the submitted work. Other relationships: All authors have declared that there are no other relationships or activities that could appear to have influenced the submitted work.

\section{Acknowledgements}

We thank Mr. Nobuyuki Sawai, Mr. Michiyuki Wada, Mr. Naonori Haraguchi, Ms. Takami Sugimoto, Ms. Yuki Suzuki, Ms. Erina Ishikawa, and Ms. Mariko Kaneko of Sano Kousei General Hospital for clinical assistance. This work was supported by Gunma University Heavy Ion Medical Center.

\section{References}

1. Ogawa Y, Takahashi T, Kobayashi T, et al.: Mechanism of hydrogen peroxide-induced apoptosis of the human osteosarcoma cell line HS-Os-1. Int J Mol Med. 2003, 12:459-463. 10.3892/ijmm.12.4.459

2. Kariya S, Sawada K, Kobayashi T, Karashima T, Shuin T, Nishioka A, Ogawa Y: Combination treatment of hydrogen peroxide and X-rays induces apoptosis in human prostate cancer PC-3 cells. Int J Radiat Oncol Biol Phys. 2009, 75:449-54. 10.1016/j.ijrobp.2009.04.092

3. Akima R, Ogawa Y, Morita-Tokuhiro S, et al.: New enzyme-targeting radiosensitizer (KORTUC) containing hydrogen peroxide \& sodium hyaluronate for intra-tumoral injection using mice transplanted with SCC VII tumor. Int J Cancer Clin Res. 2016, 3:1-6. 10.23937/2378-3419/3/2/1048

4. Nimalasena S, Gothard L, Anbalagan S, et al.: Intratumoral hydrogen peroxide with radiation therapy in locally advanced breast cancer: results from a phase 1 clinical trial. Int J Radiat Oncol Biol Phys. 2020, 108:1019-29. 10.1016/j.ijrobp.2020.06.022

5. Vaupel P, Briest S, Höckel M: Hypoxia in breast cancer: pathogenesis, characterization and biological/therapeutic implications. Wien Med Wochenschr. 2002, 152:334-42. 10.1046/j.1563258x.2002.02032.x

6. Ogawa Y, Ue H, Tsuzuki K, et al.: New radiosensitization treatment (KORTUC I) using hydrogen peroxide solution-soaked gauze bolus for unresectable and superficially exposed neoplasms. Oncol Rep. 2008, 19:1389-94. 10.3892/or.19.6.1389

7. Galluzzi L, Buqué A, Kepp O, Zitvogel L, Kroemer G: Immunogenic cell death in cancer and infectious disease. Nat Rev Immunol. 2017, 17:97-111. 10.1038/nri.2016.107

8. Gough DR, Cotter TG: Hydrogen peroxide: a Jekyll and Hyde signalling molecule . Cell Death Dis. 2011, 2:e213. 10.1038/cddis.2011.96

9. Veal EA, Day AM, Morgan BA: Hydrogen peroxide sensing and signaling. Mol Cell. 2007, 26:1-14. 10.1016/j.molcel.2007.03.016

10. Sies H: Hydrogen peroxide as a central redox signaling molecule in physiological oxidative stress: oxidative eustress. Redox Biol. 2017, 11:613-9. 10.1016/j.redox.2016.12.035 